\title{
Favourable prognostic role of histological regression in stage III positive sentinel lymph node melanoma patients
}

\author{
D Zugna ${ }^{1}$, R Senetta ${ }^{2}$, S Osella-Abate ${ }^{2}$, M T Fierro ${ }^{3}$, A Pisacane ${ }^{4}$, A Zaccagna $^{5}$, A Sapino ${ }^{4}$, V Bataille ${ }^{6,7}$,
} A Maurichi ${ }^{8}$, F Picciotto9 ${ }^{9}$ P Cassoni ${ }^{2}, \mathrm{P}$ Quaglino ${ }^{3}$ and S Ribero ${ }^{\star, 3}$

${ }^{1}$ Department of Medical Sciences, Unit of Cancer Epidemiology, CERMS, University of Turin, C.So Dogliotti, 14, Torino 10126, Italy; ${ }^{2}$ Department of Medical Sciences, Section of Surgical Pathology, University of Turin, C.So Dogliotti, 14, Torino 10126, Italy; ${ }^{3}$ Department of Medical Sciences, Section of Dermatology, University of Turin, C.So Dogliotti, 14, Torino 10126, Italy; ${ }^{4}$ Pathology Unit, Fondazione del Piemonte per I'Oncologia (FPO), Candiolo Cancer Institute (IRCCS), Km 3,95, SP142, 10060 Candiolo, Torino Italy; ${ }^{5}$ Dermatologic Surgery Section, Fondazione del Piemonte per I'Oncologia (FPO), Candiolo Cancer Institute (IRCCS), Km 3,95, SP142, 10060 Candiolo, Torino, Italy; ${ }^{6}$ Mount Vernon Cancer Centre, Rickmansworth Road, Northwood HA6 2RN, UK; ${ }^{7}$ Department of Twin Research and Genetic Epidemiology, King's College London, South Wing Block D, Westminster Bridge Road, London SE1 7EH, UK; ${ }^{8}$ Melanoma and Sarcoma Surgery Unit, Fondazione IRCCS Istituto Nazionale Tumouri, Via Giacomo Venezian, 1, 20133 Milan, Italy and ${ }^{9}$ Dermatologic Surgery Section, Department of Oncology, AOU Città della Salute e della Scienza di Torino, Via Cherasco 23, 10123 Torino, Italy

Background: Sentinel lymph node (SLN)-positive melanoma patients are a heterogeneous group of patients with survival rates ranging from $\sim 20$ to over $80 \%$. No data are reported concerning the role of histological regression on survival in stage III melanoma.

Methods: The study included 365 patients with positive SLN from two distinct hospitals. The model was developed on patients from 'AOU Città della Salute e della Scienza di Torino', and externally validated on patients from IRCCS of Candiolo. Survival analyses were carried out according to the presence of regression and adjusted for all other prognostic factors.

Results: Among patients followed at 'AOU Città della Salute e della Scienza di Torino' $(n=264)$, the median follow-up time to death or censoring (whatever two events occurred earlier) was 2.7 years since diagnosis (interquartile range: 1.3-5.8). In all, 79 patients died from melanoma and 11 from other causes. Histological regression $(n=43)$ was associated with a better prognosis (sub-HR=0.34, Cl 0.12-0.92), whereas the other factors above showed an inverse association. In the external validation, the concordance index was 0.97 at 1 year and decreased to 0.66 at 3 years and to 0.59 at 5 years. Adding histological regression in the prognostic model increased the discriminative ability to 0.75 at 3 years and to 0.62 at 5 years. Finally, using a cutoff of $20 \%$ for the risk of death led to a net re-classification improvement of 15 and $11 \%$ at 3 and 5 years after diagnosis, respectively.

Conclusions: Histological regression could lead to an improvement in prognostic prediction in patients with stage III-positive SLN melanoma.

*Correspondence: Dr S Ribero; E-mail: simone.ribero@unito.it

Received 25 April 2017; revised 22 September 2017; accepted 11 October 2017; published online 9 November 2017

(C) 2018 Cancer Research UK. All rights reserved 0007-0920/18 
Histological regression in primary melanoma is defined as replacement of tumour cells by lymphocytic inflammation, as well as attenuation of the epidermis and non-laminated dermal fibrosis with inflammatory cells, melanophagocytosis and telangiectasia (College of the American Pathologist Protocol, 2015).

Its prognostic role has been a matter of debate for many years. Previously, it has been considered a negative prognostic factor, as it may lead to an underestimation of thickness measurement. Agreement about the worst prognostic factor is only reported in totally regressed melanoma (Bartlett et al, 2016; Mihic-Probst et al, 2016).

Shaw et al (1989) hypothesised that the presence of metastatic melanoma in a regional lymph node might stimulate an immune response resulting in regression of the primary lesion. However, other studies proved that regression does not increase the risk of nodal metastases (Ma et al, 2012; Savoia et al, 2012). Kaur et al, (2008) considered primary regression as a positive prognostic feature in melanoma patients and showed no association with a higher risk of metastatic sentinel lymph node (SLN).

Previous studies have shown a favourable prognostic role of histological regression in stage I and II melanomas (Traves et al, 2012; Ribero et al, 2013a, b). A meta-analysis has recently demonstrated an inverse association between histological regression and SLN status (Ribero et al, 2015). No prognostic data are published in stage III melanoma patients with positive SLN. Therefore, we performed a study to asses the prognostic role of histological regression in primary tumours on overall survival in stage III melanomas with positive SLN.

\section{MATERIALS AND METHODS}

Data. Clinical data from 264 consecutive patients with a positive SLN for melanoma (Stage III at diagnosis according to AJCC (American Joint Committee on Cancer)) (Balch et al, 2009) were collected in this study. All patients were diagnosed and followed-up at the Department of Surgical Dermatology of the 'AOU Città della Salute e della Scienza di Torino' from 1 January 1999 to 31 December 2014. The study protocol was approved by the internal ethics committee. Patients were classified on the basis of AJCC criteria and treated and followed-up according to standard guidelines (Cochran et al, 2000; Garbe et al, 2010; Rossi et al, 2014). Sentinel lymph node biopsy (SLNB) was performed in the presence of: (1) melanoma of $>1 \mathrm{~mm}$ thickness or thinner in case of ulceration and/or mitotic rate $>1 / \mathrm{mm}^{2}$ (stage $\mathrm{T} 1 \mathrm{~b}$ disease); (2) other potential significant predictors of SLN positivity (as younger age, mitotic rate, vertical growth phase); and (3) histological regression of the primary tumour diagnosed between 1999 and 2008. After 2008, regression was no longer considered as an indication for SLNB because of the lack of evidence confirming the usefulness of this procedure in thin melanomas and in accordance with the European consensus-based interdisciplinary guidelines for diagnosis and treatment of melanoma patients (Garbe et al, 2010). The SLN evaluation protocol was in accordance with Cook and Di Palma (2008). The SLN tumour burden has been evaluated measuring the maximum diameter of the largest metastatic deposit in SLN/SLNs according with van Akkooi et al (2006).

Histological regression was evaluated on histological slides stained with haematoxylin and eosin from primary cutaneous melanoma tumoursby an experienced dermatopathologist in each centre. Based on our experience and literature data (Kang et al, 1993; Requena et al, 2009; Ribero et al, 2016a), the following histological criteria were considered in defining histological regression: replacement by lymphocytic inflammation or disappearance of melanoma cells in a circumscribed or more diffuse tumour area, as well as attenuation of the epidermis, dermal fibrosis associated to inflammatory cells (mainly lymphocytes), melanophages and telangiectasia (Supplementary Figure 1). These parameters define the histological regression in the CAP protocol (College of the American Pathologist Protocol, 2015). None of the evaluated lesions were associated with previous inflammatory or infectious reactions, or previous treatments, that could justify the development of primary tumour regression. Cases of whole regression were not included in the study cohorts.

The validation cohort was composed of 101 positive SLN melanoma patients diagnosed and followed-up at the IRCCS of Candiolo from the 1 January 2001 to 31 December 2014. The surgical protocol for the SLN as well as the inclusion criteria and the follow-up were the same as those applied at the Department of Surgical Dermatology of the 'AOU Città della Salute e della Scienza di Torino'.

Follow-up data were available up to the end of June 2015. Patients who were not recorded as dying by the date of last registration at the General Registration Office were censored at this date. Overall survival from melanoma was the outcome of interest and death from other causes was considered a competing risk, that is, an event whose occurrence precluded the occurrence of the event of interest.

Statistical analysis. The potential prognostic factors investigated were gender, age, year at diagnosis, site (head, trunk, arm, leg), Breslow thickness, evidence of ulceration, histological regression, histotype (superficial spreading, nodular, lentigo maligna, acral lentiginous), mitotic rate, SLN tumour burden and total positive lymph node count. Multiple imputations by chained equations (MICE) approach were performed assuming the data were missing at random (MAR) (Marshall et al, 2009; Steyerberg, 2009; White and Royston, 2009). Univariable analysis was performed nonparametrically by representing the cumulative incidence curves, that is, the probability of dying from melanoma, according to each potential prognostic factor, and by testing differences by covariates value by Gray's test (Gray, 1988). Variable selection was carried out by both backward stepwise approach based on Akaike criterion (Wood et al, 2008) and the least absolute shrinkage selection operator (LASSO) method. Multivariable analyses were conducted by the Fine and Gray model allowing to directly assess the effect of each covariate on cumulative incidence function (Fine and Gray, 1999). By modelling the sub-distribution hazard function, that is, the instantaneous risk of the event of interest given that an individual has survived until that time without any event or has had the competing event before that time, the effect of each potential prognostic factor on the sub-distribution hazard was quantified by sub-hazard ratio (sub-HR) and hence a measure of association with the cumulative incidence function was obtained. Proportionality assumption on the sub-hazard distribution scale was checked by including the interaction between time (on logarithmic scale) and each variable included in the model. Continuous variables were modelled firstly as linear and then as restricted cubic splines with three knots fixed at tertiles of their distribution. If both conditions of no evidence of nonlinear trends and no increase in the predictive performance of the model were satisfied, the model with linear terms (and then with reduced $d . f$.) was chosen. External validation was followed by internal validation because of low number of events in the validation set. Specifically, internal cross-validation was conducted by bootstrap resampling. The prediction models were trained on 100 bootstrap samples drawn with replacement of the same size as the original data. Discriminative ability over time was assessed by time-dependent concordance index, which quantifies the ability of the model to correctly rank events of interest up to well-defined times and to discriminate them from competing events. To deal with right censored data, inverse probability of censoring weighted estimator of the concordance index was used (Wolbers et al, 2014). 
Calibration, indicating agreement between observed outcomes and predictions, was represented by cumulative incidence estimate computed within percentiles of predicted risk, against the average predicted risk within the same percentiles of the events of interest at several time-points. Finally, to assess the incremental value of the regression as marker, we compared two models, with and without regression, by $\mathrm{C}$-index and the net reclassification improvement (NRI) over time (Pencina et al, 2011). The latter shows how many subjects are re-classified conditional on the outcome by adding a marker in the model and is given by the difference of the expected numbers of events reclassified upwards and downwards and the expected numbers of non-events reclassified downwards and upwards divided by the total expected cases of events and non-events.

Details on multiple imputation model, variable selection and the inverse probability of censoring weighted estimator of the concordance index are reported in Supplementary Material.

All analyses were performed using $\mathrm{R}$ version 3.2.3 (www.rproject.org).

\section{RESULTS}

Clinical data. Out of 264 patients followed-up at the Department of Surgical Dermatology of the 'AOU Città della Salute e della Scienza di Torino', 57.6\% (152 out of 264) were males with a median age of 57 years at diagnosis (interquartile range (IQR): $43-$ 68). Superficial spreading melanoma was the most common histotype (68\%), and the most common body sites were the trunk $(47 \%)$ and leg (39\%), respectively. Ulceration and histological regression were described in $102(40 \%)$ and 43 (16\%) patients, respectively. Median Breslow thickness was $3.3 \mathrm{~mm}$ (IQR: 2.0-5.0), median mitoses rate was 4.0 (IQR: 2-6), median SLN tumour burden was $1.8 \mathrm{~mm}$ (IQR: $0.6-4.1$ ) and the median positive SLN count ranged from 1 to 3 (median 1). Median number of positive lymph node considering SLN and CLND was 1 (IQR:1-2). In all, 66 (25\%) experienced first metastasis in regional skin (60) or regional lymph nodes (6) and $48(18 \%)$ in a distant site. The variable distribution was similar in patients followed at the IRCCS of Candiolo except for the presence of ulceration, mitoses rate and SLN tumour burden $\left(P<0.05\right.$ by $\chi^{2}$ test for categorical variables and by Wilcoxon test for continuous variables). Potential prognostic factors are reported in Table 1 and Supplementary Table 1.

Survival study. Among patients followed at 'AOU Città della Salute e della Scienza di Torino', the median follow-up time to death or censoring, whatever two events occurred earlier, was 2.7 years since diagnosis (IQR: 1.3-5.8); 79 patients (30\%) died from melanoma and 11 (4\%) from other causes. Among patients followed at IRCCS of Candiolo, the median follow-up time to death or censoring was 3.3 years since diagnosis (IQR: 1.4-5.9); 21 (21\%) patients died from melanoma and $5(5 \%)$ from other causes.

The prognostic model was developed on patients followed at 'AOU Città della Salute e della Scienza di Torino', excluding those with lentigo maligna melanoma and other rare histotypes of melanoma $(n=5)$. Cumulative incidence curves stratified according to each prognostic factor are represented in Figure 1. Gray's test did not highlight any difference in the probability of dying from melanoma by gender or body site $(P>0.05)$. Consistently with the crude analysis, the selection procedure led to the selection of variables for the predictive model as age at diagnosis, melanoma histotype, Breslow thickness, ulceration, histological regression, SLN tumour burden and positive lymph nodes count. In the multivariable analysis, continuous variables were modelled as linear. There was no evidence of non-proportionality when interactions between the prognostic factors and the time were

Table 1. Potential prognostic factors in the study data sets

\begin{tabular}{|c|c|c|}
\hline & $\begin{array}{c}\text { Molinette } \\
(n=264)\end{array}$ & $\begin{array}{l}\text { Candiolo } \\
(n=101)\end{array}$ \\
\hline Characteristics & $n(\%)$ & $n(\%)$ \\
\hline \multicolumn{3}{|l|}{ Gender } \\
\hline $\begin{array}{l}\text { Male } \\
\text { Female } \\
\text { Missing }\end{array}$ & $\begin{array}{c}152(57.6) \\
112(42.4) \\
0\end{array}$ & $\begin{array}{c}57(56.4) \\
44(43.6) \\
0\end{array}$ \\
\hline
\end{tabular}

Age at diagnosis (years)

\begin{tabular}{|l|c|c|}
\hline$\leqslant 40$ & $48(18.2)$ & $23(22.8)$ \\
$41-60$ & $105(39.8)$ & $36(35.7)$ \\
$>60$ & $111(42.4)$ & $42(41.6)$ \\
Missing & 0 & 0 \\
\hline
\end{tabular}

Year at diagnosis (years)

$\leqslant 2006$

2007-2009

2010-2012

$>2013$ Missing

$80(30.3)$

$64(24.2)$

$27(26.7)$

$69(26.1)$

$27(26.7)$

Body site 0

\begin{tabular}{l|c|c} 
Head & $13(4.9)$ & $11(10.9)$ \\
Trunk & $123(46.6)$ & $50(49.5)$ \\
Arm & $24(9.1)$ & $10(9.9)$ \\
Leg & $104(39.4)$ & $30(29.7)$ \\
Missing & 0 & 0
\end{tabular}

Missing

0

$25(24.7)$

$22(21.8) 0$

\section{Breslow AJCC}

2

3

4

Median (IQR) (mm)

Missing

Ulceration

No

Yes

Missing

8 (3.0)

62 (23.5)

94 (35.6)

$100(37.9)$

$3.3(2.0-5.0)$

0

$5(4.9)$

21 (20.8)

$53(52.5)$

$22(21.8)$

$3.0(2.0-4.0)$

1

\section{Histological regression}

\begin{tabular}{|l|c|c|}
\hline No & $221(83.7)$ & $80(79.2)$ \\
Yes & $43(16.3)$ & $21(20.8)$ \\
Missing & 0 & 0 \\
\hline
\end{tabular}

\section{Histotype}

Superficial spreading melanoma

Nodular melanoma

Lentigo maligna melanoma

Acral lentiginous melanoma

Other

Missing

$153(60.0)$

$102(40.0)$

$32(41.0)$

$46(59.0)$

9

23

Mitotic rate $\left(1 / \mathrm{mm}^{2}\right)$

0

$\geqslant 1$

Median (IQR)

$78(67.7)$

Missing

$49(18.6)$

$3(1.1)$

$29(11.0)$

4 (1.5)

\begin{tabular}{c|c}
$70(69.3)$ \\
$24(23.8)$ \\
$/$ \\
$7(6.9)$ \\
$/$ \\
0
\end{tabular}

\section{Positive lymph nodes}

\begin{tabular}{|l|c|c|}
\hline 1 & $148(57.1)$ & $50(54.9)$ \\
2 & $65(25.1)$ & $26(28.6)$ \\
$\geqslant 3$ & $46(17.7)$ & $9(16.5)$ \\
Median (IQR) & $1(1-2)$ & $1(1-2)$ \\
Missing & 5 & 10 \\
\hline SLN tumour burden (mm) & $11(4.4)$ & $12(12.6)$ \\
\hline 0.10 & $79(31.5)$ & $31(32.6)$ \\
$0.10-1.00$ & $161(64.1)$ & $52(54.7)$ \\
$>1.00$ & $1.8(0.6-4.1)$ & $1.4(0.4-2.5)$ \\
Median (IOR) & 13 & 6 \\
Missing & \multicolumn{2}{|c|}{} \\
\hline Abbreviations: AJCC = American Joint Committee on Cancer; IQR=interquartile range; \\
SLN=sentinel lymph node.
\end{tabular}



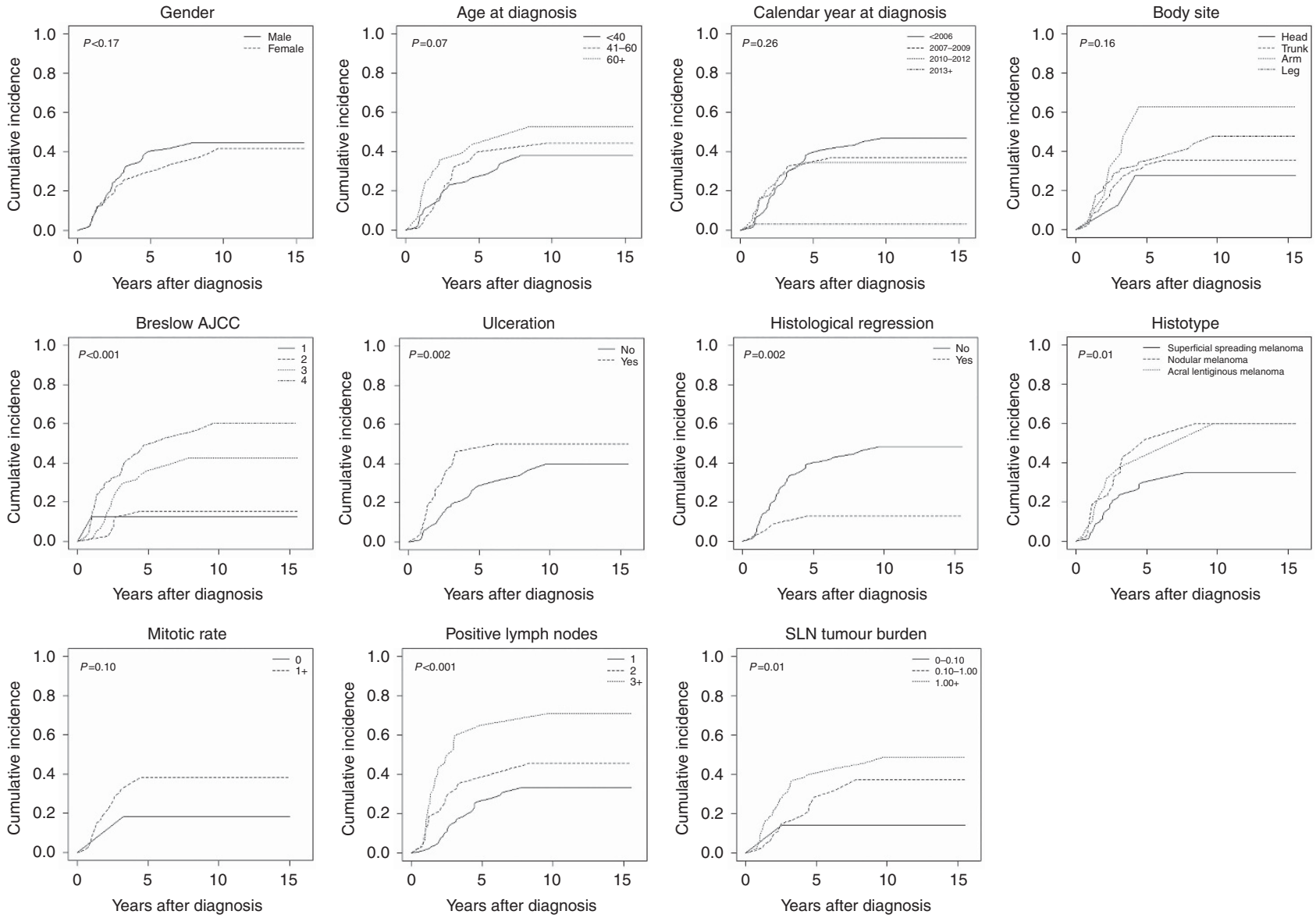

Figure 1. Non-parametric cumulative incidence curves by potential prognostic factors. P-value calculated by Gray's test.

included in the model. As the parameters estimated by Fine and Gray model measure the association between each factor and the cumulative incidence function, an increase in the risk of dying from melanoma was observed among older patients, nodular melanomas, thicker melanomas, ulcerated melanomas, higher positive lymph node count and increased SLN tumour burden (Table 2). Histological regression at diagnosis was associated with a decreased risk of death from melanoma in both univariable and multivariable analyses (Table 2). When analyses were performed on patients from IRCCS of Candiolo, the direction of the association was maintained but the confidence interval was much larger and containing the null value (sub-HR $=0.73$, 95\% CI: $0.11-$ 4.93). The $\mathrm{C}$-index, ranging from 0.5 when the model has no ability to discriminate between low- and high-risk subjects and 1 when the model perfectly discriminates between the two groups, was 0.79 at 1 year after diagnosis and decreased to 0.73 at 3 and 5 years after diagnosis in the training set. In the external validation, C-index was 0.97 at 1 year after diagnosis and decreased to 0.66 at 3 years after diagnosis and to 0.59 at 5 years after diagnosis. In the internal cross-validation, C-index was 0.74 at 1 year after diagnosis and decreased to 0.69 at 3 and 5 years after diagnosis (Figure 2) and the calibration at different time-points indicated that low predictions were slightly too low and high predictions were slightly too high (Figure 3).

Clinical implications. Predictive probability of death from melanoma at 3 and 5 years after diagnosis for 14 hypothetical clinical scenarios is reported in Supplementary Table 2. For example, the cumulative incidence at 3 and 5 years for a patient diagnosed at 65 years of age with superficial spreading melanoma, ulceration, one positive lymph node, $1 \mathrm{~mm}$ of Breslow thickness, positive SLN ( $0.5 \mathrm{~mm}$ in maximum diameter) and no evidence of histological regression was $17.1 \%$ and $25.8 \%$ respectively. It decreased to $6.1 \%$ at 3 years and to $9.6 \%$ at 5 years after diagnosis in a patient with the same characteristics as above but evidence of histological regression. When comparing the discriminative ability of the models without and with histological regression, the C-index increased from 0.70 to 0.73 at 3 and 5 years after diagnosis. In the validation set, the C-index increased from 0.66 to 0.75 and from 0.59 to 0.62 at 3 and 5 years after diagnosis, respectively. Using a cutoff of $20 \%$ for the risk of dying from melanoma led to classification of 120 and 124 patients at 3 years and 203 and 195 at 5 years after diagnosis at high risk of death from melanoma according to the model without and with histological regression, respectively. The NRI was $14.8 \%$ and $11.2 \%$ at 3 and 5 years after diagnosis, respectively.

\section{DISCUSSION}

Here we report that stage III melanoma patients, defined as having positive SLNB, showed a better prognosis when histological regression was present in the primary tumour, independently from other prognostic factors. In addition, the developed prognostic model confirms the relevance of the three classical prognostic parameters in stage III melanoma (Breslow thickness, ulceration and positive lymph nodes count) and proves that SLN tumour burden, histotype and age also bear prognostic significance.

Many parameters have previously been analysed to find out new prognostic parameters for melanoma patients. Age at diagnosis, primary cutaneous tumour site and number of lymphatic basin 
Table 2. Melanoma-specific sub-hazard ratio (sub-HR) estimated by Fine and Gray model with $95 \%$ confidence interval $(95 \% \mathrm{CI})$ in 'Città della Salute e della Scienza di Torino

\begin{tabular}{|c|c|c|c|c|}
\hline \multirow[b]{2}{*}{ Variable } & \multicolumn{2}{|c|}{ Univariable } & \multicolumn{2}{|c|}{ Multivariable } \\
\hline & Sub-HR & $95 \% \mathrm{Cl}$ & $\begin{array}{c}\text { Sub- } \\
\text { HR }\end{array}$ & $95 \% \mathrm{Cl}$ \\
\hline \multicolumn{5}{|l|}{ Gender } \\
\hline Male & 1.00 & Ref. & I & / \\
\hline Female & 0.80 & $0.50-1.27$ & / & / \\
\hline \multicolumn{5}{|l|}{ Age at diagnosis } \\
\hline Unit increase (year) & 1.02 & $1.00-1.04$ & 1.01 & $1.00-1.03$ \\
\hline \multicolumn{5}{|l|}{ Year at diagnosis } \\
\hline Unit increase (year) & 0.98 & $0.92-1.03$ & I & I \\
\hline \multicolumn{5}{|l|}{ Body site } \\
\hline Head & 1.00 & Ref. & / & / \\
\hline Trunk & 1.43 & $0.38-5.39$ & I & I \\
\hline Arm & 2.64 & $0.65-10.74$ & I & / \\
\hline Leg & 1.85 & $0.50-6.92$ & I & I \\
\hline \multicolumn{5}{|l|}{ Breslow } \\
\hline Unit increase $(\mathrm{mm})$ & 1.22 & $1.13-1.30$ & 1.13 & $1.04-1.22$ \\
\hline \multicolumn{5}{|l|}{ Ulceration } \\
\hline No & 1.00 & Ref. & 1.00 & Ref. \\
\hline Yes & 1.95 & $1.24-3.06$ & 1.22 & $0.73-2.03$ \\
\hline \multicolumn{5}{|c|}{ Histological regression } \\
\hline No & 1.00 & Ref. & 1.00 & Ref. \\
\hline Yes & 0.26 & $0.09-0.71$ & 0.34 & $0.12-0.92$ \\
\hline \multicolumn{5}{|l|}{ Histotype } \\
\hline $\begin{array}{l}\text { Superficial spreading } \\
\text { melanoma }\end{array}$ & 1.00 & Ref. & 1.00 & Ref. \\
\hline Nodular melanoma & 2.00 & $1.22-3.29$ & 1.38 & $0.82-2.32$ \\
\hline $\begin{array}{l}\text { Acral lentiginous } \\
\text { melanoma }\end{array}$ & 1.70 & $0.85-3.40$ & 0.63 & $0.27-1.45$ \\
\hline \multicolumn{5}{|l|}{ Mitotic rate } \\
\hline Unit increase $\left(1 / \mathrm{mm}^{2}\right)$ & 1.04 & $0.99-1.08$ & I & I \\
\hline \multicolumn{5}{|c|}{ Positive lymph nodes } \\
\hline Unit increase & 1.40 & $1.26-1.57$ & 1.28 & $1.15-1.44$ \\
\hline \multicolumn{5}{|l|}{ SLN tumour burden } \\
\hline Unit increase $(\mathrm{mm})$ & 1.09 & $1.05-1.12$ & 1.04 & $1.00-1.09$ \\
\hline
\end{tabular}

drainage have been proposed even if they have not been included yet in the AJCC classification (Balch et al, 2013; Pasquali et al, 2014; Sanlorenzo et al, 2015).

Histological regression in primary cutaneous melanoma occurs in 10-35\% of cases (Blessing et al, 1990; Sanlorenzo et al, 2015). In our series, regression was found in $16 \%$ of stage III melanoma patients. Histological regression has traditionally been considered as a marker of poor prognosis, mainly in thin melanomas, as it hampers a real evaluation of the initial thickness of the tumour. However, the majority of these studies are based on small series of patients, and have been performed in single institutions (Ronan et al, 1987; Slingluff and Seigler, 1992; Ribero et al, 2016b). The introduction of SLNB has changed the clinical approach to melanoma patients in the past 20 years (Morton et al, 2006) A recent metaanalysis in more than 10000 patients eligible for SLN biopsy showed that SLN status is inversely correlated with histological regression (Ribero et al, 2015): therefore, histological regression seems to be inversely associated with the risk of lymph node metastases.

A study on 1600 patients in stage I-II melanoma showed a protective role of histological regression on survival despite all adjustments (Ribero et al, 2013b), and confirms results previously

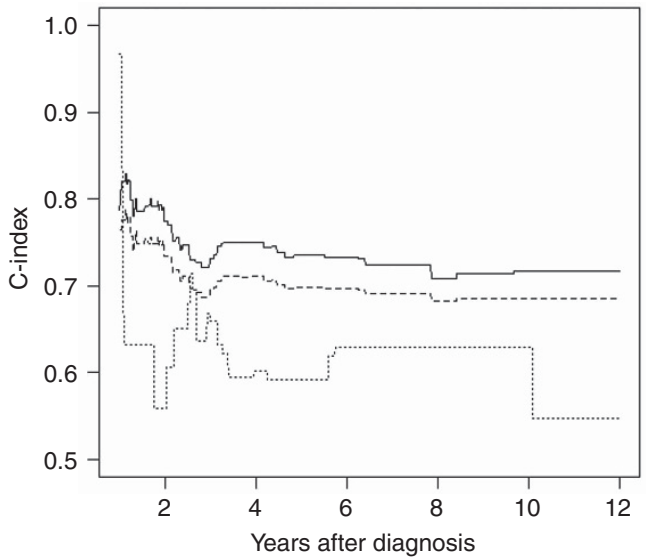

Figure 2. C-index in the training set (solid line), in the internal validation set (dash line), and in the external validation set (dotted line) over time.

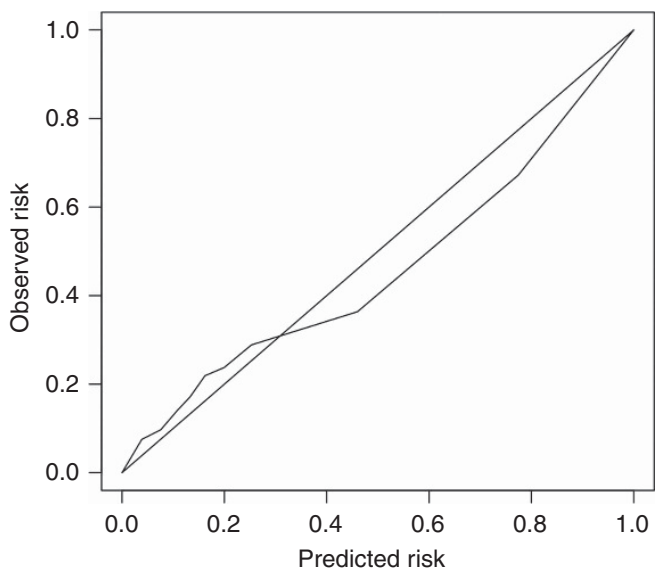

Figure 3. Plot of cumulative incidence estimate computed within percentiles of predicted risk, against the average predicted risk within the same percentiles of the events of interest at 3 years after diagnosis.

reported by other authors through a univariable analyses. Similarly, a recent meta-analysis on survival of $>8500$ melanoma patients reported a lower relative risk of death (RR $0.772,95 \% \mathrm{CI}$, 0.612-0.973) for patients with histological regression than patients without (Gualano et al, 2017). A host immunological response to the primary tumour is presumed to be at the basis of histological regression, and likely reflects an active immunological response considered as prognostically favourable. In fact, Ma et al, (2012) reported that the presence of primary tumour histological regression results from a $\mathrm{T}$ cell immune response. No data had been reported on the role of histologic regression in the stratification of survival in stage III melanoma patients. The reported prognostic model in the study reported here was developed and externally validated using two distinct Italian cohorts of 264 and 101 patients diagnosed at stage III melanoma with positive SNL. These patients were followed-up in two hospitals adopting the same SLN surgical and follow-up protocols. The prognostic model was formulated by considering the main aspects of survival data, right censoring and competing risks. The former was taken into account by including appropriate weights in calculating the model's performance and the latter was considered by indirectly modelling cumulative incidence function. Model's performance, measured by time-dependent C-index and calibration plot, was discrete in the first years after diagnosis but then worsened over time when fewer events were observed due to short 
follow-up of patients. However, the inclusion of histological regression in the prognostic model increased its discriminative ability in both the training and validation sets over time and led to a reclassification of $15 \%$ and $11 \%$ of patients, respectively, at 3 and 5 years after diagnosis when the cutoff for the risk of dying from melanoma was fixed at $20 \%$. Hence, the inclusion of histological regression in the risk equation may lead to more precise classification of patients' risk of death, helping in the decisional process for a clinical setting in stage III melanoma patients.

Because of the relatively recent (1999) introduction of the SLNB technique in both institutions in this study, the follow-up time is relatively short. This could affect the number of observed events and consequently the power of the study. In particular, the predictive ability of the model was affected, in terms of magnitude and high variability, by the small size of validation set.

In conclusion, this study demonstrates that histological regression is independently associated with a lower death rate in stage III melanoma patients and that its inclusion in the prognostic model may improve the prognostic classification of patients at higher risk of dying from melanoma. Further research is required to improve the model's performance over longer follow-up.

\section{ACKNOWLEDGEMENTS}

This study was partially funded by Lanzavecchia-Lastretti Foundation for 'Progetto Melanoma' (Rebecca Senetta). The funders had no role in study design, data collection and analyses, decision to publish or preparation of the manuscript.

\section{CONFLICT OF INTEREST}

The authors declare no conflict of interest.

\section{REFERENCES}

Balch CM, Gershenwald JE, Soong SJ, Thompson JF, Atkins MB, Byrd DR, Buzaid AC, Cochran AJ, Coit DG, Ding S, Eggermont AM, Flaherty KT, Gimotty PA, Kirkwood JM, McMasters KM, Mihm Jr MC, Morton DL, Ross MI, Sober AJ, Sondak VK (2009) Final version of 2009 AJCC melanoma staging and Classification. J Clin Oncol 27(36): 6199-6206.

Balch CM, Soong SJ, Gershenwald JE, Thompson JF, Coit DG, Atkins MB, Ding S, Cochran AJ, Eggermont AM, Flaherty KT, Gimotty PA, Johnson TM, Kirkwood JM, Leong SP, McMasters KM, Mihm Jr MC, Morton DL, Ross MI, Sondak VK (2013) Age as a prognostic factor in patients with localized melanoma and regional metastases. Ann Surg Oncol 20(12): 3961-3968.

Bartlett EK, Peters MG, Blair A, Etherington MS, Elder DE, Xu XG, Guerry D, Ming ME, Fraker DL, Czerniecki BJ, Gimotty PA, Karakousis GC (2016) Identification of patients with intermediate thickness melanoma at low risk for sentinel lymph node positivity. Ann Surg Oncol 23(1): 250-256.

Blessing K, McLaren KM, McLean A, Davidson P (1990) Thin malignant melanomas (less than $1.5 \mathrm{~mm}$ ) with metastasis: a histological study and survival analysis. Histopathology 17(5): 389-395.

Cochran AJ, Balda BR, Starz H, Bachter D, Krag DN, Cruse CW, Pijpers R, Morton DL (2000) The Augsburg consensus. Techniques of lymphatic mapping, sentinel lymphadenectomy, and completion lymphadenectomy in cutaneous malignancies. Cancer 89: 236-241.

College of the American Pathologist (2015) Protocol for the Examination of Specimens from Patients with Melanoma of the Skin. Based on AJCC/ UICC TNM, 7th edition. Protocol web posting date: February.

Cook MG, Di Palma S (2008) Pathology of sentinel lymph nodes for melanoma. J Clin Pathol 61(8): 897-902.

Fine JP, Gray RJ (1999) A proportional hazards model for the subdistribution of a competing risk. J Am Stat Assoc 94: 496-509.

Garbe C, Peris K, Hauschild A, Saiag P, Middleton M, Spatz A, Grob JJ, Malvehy J, Newton-Bishop J, Stratigos A, Pehamberger H, Eggermont A
(2010) Diagnosis and treatment of melanoma: European consensus-based interdisciplinary guideline. Eur J Cancer 46(2): 270-283.

Gray RJ (1988) A class of K-sample tests for comparing the cumulative incidence of a competing risk. Ann Stat 16: 1141-1154.

Gualano MR, Osella-Abate S, Scaioli G, Marra E, Bert F, Faure E, Sportoletti Baduel E, Balagna E, Quaglino P, Fierro MT, Siliquini R, Ribero S (2017) Prognostic role of histologic regression in primary cutaneous melanoma: a systematic review and meta-analysis. Br J Dermatol.

Kang S, Barnhill RL, Mihm Jr MC, Sober AJ (1993) Histologic regression in malignant melanoma: an interobserver concordance study. J Cutan Pathol 20: $126-129$.

Kaur C, Thomas RJ, Desai N, Green MA, Lovell D, Powell BW, Cook MG (2008) The correlation of regression in primary melanoma with sentinel lymph node status. J Clin Pathol 61(3): 297-300.

Ma MW, Medicherla RC, Qian M, Vega-Saenz de Miera E, Friedman EB, Berman RS, Shapiro RL, Pavlick AC, Ott PA, Bhardwaj N, Shao Y, Osman I, Darvishian F (2012) Immune response in melanoma: an indepth analysis of the primary tumor and corresponding sentinel lymph node. Mod Pathol 25(7): 1000-1010.

Marshall A, Altman DG, Holder RL, Royston P (2009) Combining estimates of interest in prognostic modelling studies after multiple imputation: current practice and guidelines. BMC Med Res Methodol 9: 57.

Mihic-Probst D, Shea C, Duncan L, de la Fouchardiere A, Landman G, Landsberg J, ven den Oord J, Lowe L, Cook MG, Yun SJ, Clarke L, Messina J, Elder DE, Barnhill RL (2016) Update on thin melanoma: outcome of an International Workshop. Adv Anat Pathol 23(1): 24-29.

Morton DL, Thompson JF, Cochran AJ, Mozzillo N, Elashoff R, Essner R, Nieweg OE, Roses DF, Hoekstra HJ, Karakousis CP, Reintgen DS, Coventry BJ, Glass EC, Wang HJ, MSLT Group (2006) Sentinel-node biopsy or nodal observation in melanoma. N Engl J Med 355(13): 1307-1317.

Pasquali S, Mocellin S, Mozzillo N, Maurichi A, Quaglino P, Borgognoni L, Solari N, Piazzalunga D, Mascheroni L, Giudice G, Patuzzo R, Caracò C, Ribero S, Marone U, Santinami M, Rossi CR (2014) Nonsentinel lymph node status in patients with cutaneous melanoma: results from a multiinstitution prognostic study. J Clin Oncol 32(9): 935-941.

Pencina MJ, Steyerberg EW, D’Agostino RB (2011) Extensions of net reclassification improvement calculations to measure usefulness of new biomarkers. Stat Med 30(1): 11-21.

Requena C, Botella-Estrada R, Traves V, Nagore E, Almenar S, Guillén C (2009) Problems in defining melanoma regression and prognostic implication. Acta Dermosifiliogr 100: 759-766.

Ribero S, Moscarella E, Ferrara G, Piana S, Argenziano G, Longo C (2016a) Regression in cutaneous melanoma: a comprehensive review from diagnosis to prognosis. J Eur Acad Dermatol Venereol 30(12): 2030-2037.

Ribero S, Osella-Abate S, Pasquali S, Rossi CR, Borgognoni L, Piazzalunga D, Solari N, Schiavon M, Brandani P, Ansaloni L, Ponte E, Silan F, Sommariva A, Bellucci F, Macripò G, Quaglino P (2016b) Prognostic role of multiple lymphatic basin drainage in sentinel lymph node-negative trunk melanoma patients: a multicenter study from the Italian melanoma intergroup. Ann Surg Oncol 23(5): 1708-1715.

Ribero S, Quaglino P, Osella-Abate S, Sanlorenzo M, Senetta R, Macrì L, Savoia P, Macripò G, Sapino A, Bernengo MG (2013a) Relevance of multiple basin drainage and primary histologic regression in prognosis of trunk melanoma patients with negative sentinel lymph nodes. J Eur Acad Dermatol Venereol 27(9): 1132-1137.

Ribero S, Osella-Abate S, Sanlorenzo M, Savoia P, Astrua C, Cavaliere G, Tomasini C, Senetta R, Macripò G, Bernengo MG, Quaglino P (2013b) Favourable prognostic role of regression of primary melanoma in AJCC stage I-II patients. Br J Dermatol 169(6): 1240-1245.

Ribero S, Gualano MR, Osella-Abate S, e Scaioli G, Bert F, Sanlorenzo M, Balagna E, Fierro MT, Macripò G, Sapino A, Siliquini R, Quaglino P (2015) Association of histologic regression in primary melanoma with sentinel lymph node status: a systematic review and meta-analysis. JAMA Dermatol 151(12): 1301-1307.

Ronan SG, Eng AM, Briele HA, Shioura NN, Das Gupta TK (1987) Thin malignant melanomas with regression and metastases. Arch Dermatol 123(10): $1326-1330$.

Rossi CR, Mozzillo N, Maurichi A, Pasquali S, Macripò G, Borgognoni L, Solari N, Piazzalunga D, Mascheroni L, Giudice G, Mocellin S, Patuzzo R, Caracò C, Ribero S, Marone U, Santinami M (2014) Number of excised lymph nodes as a quality assurance measure for lymphadenectomy in melanoma. JAMA Surg 149(7): 700-706. 
Sanlorenzo M, Osella-Abate S, Ribero S, Marenco F, Nardò T, Fierro MT, Novelli M, Cervetti O, Bernengo MG, Quaglino P (2015) Melanoma of the lower extremities: foot site is an independent risk factor for clinical outcome. Int J Dermatol 54(9): 1023-1029.

Savoia P, Fava P, Caliendo V, Osella-Abate S, Ribero S, Quaglino P, Macripò G, Bernengo MG (2012) Disease progression in melanoma patients with negative sentinel lymph node: does false-negative specimens entirely account for this phenomenon? J Eur Acad Dermatol Venereol 26(2): 242-248.

Shaw HM, McCarthy SW, McCarthy WH, Thompson JF, Milton GW (1989) Thin regressing malignant melanoma: significance of concurrent regional lymph node metastases. Histopathology 15(3): 257-265.

Slingluff Jr CL, Seigler HF (1992) 'Thin' malignant melanoma: risk factors and clinical management. Ann Plast Surg 28(1): 89-94.

Steyerberg EW (2009) Clinical Prediction Models. Springer: New York.

Traves V, Botella-Estrada R, Requena C, Nagore E (2012) Regression does not significantly underestimate melanoma thickness. Melanoma Res 22(1): 96-98. van Akkooi AC, de Wilt JH, Verhoef C, Schmitz PI, van Geel AN, Eggermont AM, Kliffen M (2006) Clinical relevance of melanoma micrometastases $(<0.1 \mathrm{~mm})$ in sentinel nodes: are these nodes to be considered negative? Ann Oncol 17(10): 1578-1585.

White IR, Royston P (2009) Imputing missing covariate values for the Cox model. Stat Med 28(15): 1982-1998.

Wolbers M, Blanche P, Koller MT, Witteman JC, Gerds TA (2014) Concordance for prognostic models with competing risks. Biostatistics 15(3): 526-539.

Wood AM, White IR, Royston P (2008) How should variable selection be performed with multiply imputed data? Stat Med 27: 3227-3246.

This work is published under the standard license to publish agreement. After 12 months the work will become freely available and the license terms will switch to a Creative Commons AttributionNonCommercial-Share Alike 4.0 Unported License.

Supplementary Information accompanies this paper on British Journal of Cancer website (http://www.nature.com/bjc) 